\title{
A Holographic Approach to Non-Relativistic Logarithmic CFTs
}

\author{
Wout Merbis** \\ University of Groningen \\ E-mail: w.merbis@rug.nl
}

\begin{abstract}
We construct a non-relativistic scaling version of a Logarithmic Conformal Field Theory at the level of two-point correlation functions. This non-relativistic LCFT is defined holographically by considering a scalar field model as a toy model of critical gravity, in a fixed Lifshitz background.
\end{abstract}

Proceedings of the Corfu Summer Institute 2011 "School and Workshops on Elementary Particle Physics and Gravity"

September 4-18, 2011

Corfu, Greece

\footnotetext{
* Speaker.

${ }^{\dagger}$ Based on [1] with E.A. Bergshoeff, J. Rosseel and S. de Haan
} 


\section{Introduction}

Gauge/gravity dualities have become an important approach into studying both quantum field theories and quantum gravitation from a novel perspective. The most studied duality is the AdS/CFT correspondence, which relates type IIB string theory on $\mathrm{AdS}_{5} \times \mathrm{S}^{5}$ to the four dimensional superconformal field theory $\mathscr{N}=4$ SYM [2]. Over the years this correspondence has been generalized in order to make a connection to more physical systems, like the quark-gluon plasma or condensed matter systems. The strong-weak nature of the duality allows for a description of strongly coupled quantum field theories in terms of higher dimensional classical gravity. This is especially promising, as it can provide us with a tool for understanding strongly coupled quantum field theories. Here we will focus on two developments in this field of research which have been made independently: the generalization of the AdS/CFT correspondence to systems with anisotropic scale invariance $[3,4,5]$ and the AdS/logarithmic CFT correspondence for theories of massive gravity $[6,7,8,9,10,11,12,13,14,15]$.

Motivated by applications in condensed matter physics an anisotropically scaling version of the AdS/CFT correspondence has been proposed. This anisotropy is characterized by a dynamical exponent $z$, which determines how scale transformations act differently on the spatial and the time coordinates; $x \rightarrow \lambda x, t \rightarrow \lambda^{z} t$. Such a symmetry is generated by the Lifshitz algebra, which consists of spatial rotations $M_{i j}$ and translations $P_{i}$, time translations $H$ and a scaling transformation $D$. Their commutation relations are as usual, except for the scaling transformation which acts differently on $P_{i}$ and $H$ :

$$
\left[D, M_{i j}\right]=0, \quad\left[D, P_{i}\right]=i P_{i}, \quad[D, H]=i z H .
$$

The Lifshitz algebra is realized geometrically by the Lifshitz metric [3]:

$$
d s_{\mathrm{Lif}_{\mathrm{d}+1}}^{2}=L^{2}\left(\frac{1}{r^{2 z}} d t^{2}+\frac{1}{r^{2}} d r^{2}+\frac{1}{r^{2}} d x^{a} d x_{a}\right),
$$

with $a=1, \ldots d-1$. The isometries of this metric are precisely the symmetries of the Lifshitz algebra. Since this algebra gives rise to non-relativistic dispersion relations, these types of gauge/gravity dualities are often referred to as non-relativistic holography.

Another development in gauge/gravity dualities is the connection between Logarithmic Conformal Field Theories (LCFTs) and theories of massive gravity at a critical point in their parameter space (i.e. Log gravity and critical gravity). Generically these theories have massive and massless spin-2 excitations in their spectrum. At the critical point the massive excitations become massless and degenerate with the massless Einstein-Hilbert spin-2 mode. Due to this degeneracy a new mode with logarithmic falloff behavior appears. In the next section the connection between the log-modes and LCFTs will be reviewed in more detail. Let us here comment briefly on the nature of LCFTs and their difference from 'ordinary' CFTs. For a more detailed review on LCFTs and references we refer to $[16,17,18]$.

Conformal Field Theories are invariant under scale transformations that preserve angles. The two-point correlation functions are restricted by conformal symmetry to behave like:

$$
\left\langle\mathscr{O}_{i}(x) \mathscr{O}_{j}(y)\right\rangle=\delta_{i j} \frac{c}{|x-y|^{2 \Delta}},
$$


and there exists an Hamiltonian that is diagonalizable:

$$
\left[H, \mathscr{O}_{i}\right]=E_{0} \mathscr{O}_{i} .
$$

Logarithmic CFT's arise when two operators degenerate in all quantum numbers. The two operators will form an indecomposable, but non-irreducible representation of the CFT symmetry algebra (Jordan cells). The Hamiltonian no longer acts diagonally on the operators, but instead:

$$
\left[H, \mathscr{O}_{i}^{\log }\right]=E_{0} \mathscr{O}_{i}^{\log }+\mathscr{O}_{i}, \quad\left[H, \mathscr{O}_{i}\right]=E_{0} \mathscr{O}_{i},
$$

where $\mathscr{O}_{i}^{\log }$ is called the logarithmic partner of $\mathscr{O}_{i}$. The two point correlation functions will now involve logarithmic terms:

$$
\begin{gathered}
\left\langle\mathscr{O}_{i}(x) \mathscr{O}_{j}(y)\right\rangle=0, \\
\left\langle\mathscr{O}_{i}^{\log }(x) \mathscr{O}_{j}(y)\right\rangle=\delta_{i j} \frac{b}{|x-y|^{2 \Delta}}, \\
\left\langle\mathscr{O}_{i}^{\log }(x) \mathscr{O}_{j}^{\log }(y)\right\rangle=\delta_{i j} \frac{1}{|x-y|^{2 \Delta}}(-2 b \log |x-y|+\lambda) .
\end{gathered}
$$

The rank of the LCFT determines the amount of logarithmic partner operators. Typically this can be any integer and the correlation function will show higher power log-terms. In this work we will limit the discussion to rank 2 , where there is one null state, corresponding to (1.6), and one logarithmic partner.

The outline of the remainder of this paper is as follows. In section 2 we will devote some attention to the connection between critical gravity and LCFTs. Then, in section 3 we will introduce a simple scalar field model, similar to the ones discussed in $[19,20]$ which shares its general structure with critical gravity. When considering this model in a fixed Lifshitz background, we define a nonrelativistic version of an LCFT by calculating its two-point correlation functions holographically. Finally, we conclude in the last section.

\section{AdS/LCFT from Critical Gravity}

Einstein-Hilbert gravity with a cosmological constant may be supplemented with terms which are higher order in derivatives of the metric. This results in a class of theories of massive gravity. The original motivation for studying these theories was to improve the renormalizability of gravity, however it soon became apparent these theories of massive gravity have problems with unitarity.

In three dimensions, the most general fourth order theory of massive gravity which does not propagate scalar modes is Generalized Massive Gravity (GMG). It's Lagrangian is given by:

$$
\mathscr{L}_{\mathrm{GMG}}=\sigma R-2 \lambda m^{2}+\frac{1}{\mu} \mathscr{L}_{\mathrm{LCS}}+\frac{1}{m^{2}} \mathscr{L}_{\mathrm{R}^{2}}
$$

with $\sigma= \pm 1$ and $\lambda$ is a dimensionless cosmological parameter. $\mathscr{L}_{\mathrm{LCS}}$ is the topological LorentzChern-Simons term:

$$
\mathscr{L}_{\mathrm{LCS}}=\varepsilon^{\mu v \rho} \Gamma_{\mu \alpha}^{\gamma}\left(\partial_{v} \Gamma_{\rho \gamma}^{\alpha}+\frac{2}{3} \Gamma_{v \beta}^{\alpha} \Gamma_{\rho \gamma}^{\beta}\right),
$$

and the curvature squared term is given by:

$$
\mathscr{L}_{\mathrm{R}^{2}}=R_{\mu v} R^{\mu \nu}-\frac{3}{8} R^{2}
$$


In the limit $m^{2} \rightarrow \infty$, while keeping $\lambda m^{2}=-1 / l^{2}$ fixed, the Lagrangian (2.1) reduces to Topological Massive Gravity (TMG) [21], which propagates a single massive mode of helicity 2 . At the special value of the parameters $\mu l=1$ the massive mode becomes massless. The remaining theory with Fefferman-Graham-Brown-Henneaux boundary conditions (which are asymptotically $\mathrm{AdS}_{3}$ ) is called chiral gravity [22, 23]. It has been shown that this theory is chiral and has positive energy solutions (despite of suffering from linearization instabilities). If one relaxes the Brown-Henneaux boundary conditions to allow for logarithmic fall-off behavior, one obtains Log gravity. The theory is no longer chiral and it is non-unitary, but the upshot is that its dual CFT is a Logarithmic CFT (which is also non-unitary) [7, 8, 23].

In the remainder of this paper we focus on another limit in (2.1): $\mu \rightarrow \infty$. In that case the Lagrangian reduces to that of New Massive Gravity (NMG) [24]. In contrast to TMG, NMG is parity even and propagates two massive modes with helicities \pm 2 . In three dimensions it is perturbatively unitary for a 'wrong sign' Einstein-Hilbert term $(\sigma=-1)$. Unfortunately, for this value of $\sigma$ the BTZ black hole has negative mass, so the full non-linear theory is not unitary.

Unlike TMG, New Massive Gravity can be extended to $d$ dimensions. Its action is:

$$
S=\frac{1}{\kappa^{2}} \int d^{d} x \sqrt{-g}\left\{\sigma R-2 \lambda m^{2}+\frac{1}{(d-2)} f^{\mu v} G_{\mu v}-\frac{m^{2}}{4(d-2)}\left(f^{\mu v} f_{\mu v}-f^{2}\right)\right\},
$$

where the 'auxiliary field' $f_{\mu \nu}$ is a symmetric two-tensor that is used to lower the number of derivatives in the action from four to two. After linearizing this action around an $\mathrm{AdS}_{d}$ background the Lagrangian reduces to [13]:

$$
\mathscr{L}_{2}=-\frac{1}{2} \bar{\sigma} h^{\mu v} \mathscr{G}_{\mu v}(h)+\frac{2}{m^{2}(d-1)(d-2)} k^{\mu v} \mathscr{G}_{\mu v}(k)-\frac{2}{m^{2}(d-1)^{2}(d-2)}\left(k^{\mu v} k_{\mu v}-k^{2}\right) .
$$

Here $h, k$ are the fluctuations of the metric and the auxiliary field tensor respectively. The parameter $\bar{\sigma}$ is given by:

$$
\bar{\sigma}=\sigma-\frac{\lambda}{m^{2}} \frac{1}{d-1} .
$$

Depending on the value of $\bar{\sigma}$ one can distinguish two case:

- $\bar{\sigma} \neq 0 \Rightarrow$ non-critical gravity

- $\bar{\sigma}=0 \Rightarrow$ critical gravity

We will now briefly discuss both cases. The linearized equations of motion of NMG can be reduced to:

$$
\left(\square-\frac{4 \Lambda}{(d-1)(d-2)}-M^{2}\right)\left(\square-\frac{4 \Lambda}{(d-1)(d-2)}\right) h_{\mu \nu}=0,
$$

with:

$$
M^{2}=-m^{2}(d-2) \bar{\sigma} .
$$

From these equations it is clear that the spectrum contains a massive and a massless mode. The massive mode $h_{\mu \nu}^{\mathrm{M}}$ solves the equation $\left(\square-\frac{4 \Lambda}{(d-1)(d-2)}-M^{2}\right) h_{\mu \nu}^{\mathrm{M}}=0$, while the massless mode $h_{\mu \nu}^{\mathrm{m}}$ is a solution of $\left(\square-\frac{4 \Lambda}{(d-1)(d-2)}\right) h_{\mu \nu}^{\mathrm{m}}=0$. 
In three dimensions, the massless excitations do not propagate in the bulk and only describe boundary gravitons. The only propagating bulk modes come from the $k$-fluctuations which are described by a Fierz-Pauli Lagrangian with a mass term. One is thus allowed to choose the sign in front of the massless term in such a way that both modes have positive energy. This is achieved for $\sigma=-1$.

For dimensions $d>3$ both modes propagate, while their energies have opposite sign [10], except at the critical point where $\frac{\lambda}{m^{2}} \frac{1}{d-1}=\sigma$. At this point the energies of both modes vanish (as well as the energy of the Schwarzschild-AdS black hole). In addition the mass (2.8) vanishes and the massive modes degenerate with the massless modes. Now a new solution $h_{\mu \nu}^{\log }$ can be found, which obeys the equation of motion:

$$
\left(\square-\frac{4 \Lambda}{(d-1)(d-2)}\right)^{2} h_{\mu \nu}^{\log }=0,
$$

but it is not annihilated by acting with the operator in the parenthesis only once. Instead we have that:

$$
\left(\square-\frac{4 \Lambda}{(d-1)(d-2)}\right) h_{\mu \nu}^{\log }=h_{\mu \nu}^{\mathrm{m}} .
$$

This is the logarithmic mode and it's characterized by a logarithmic fall off behavior to the boundary. An explicit solution for the log-mode has been found in $d=4$ for global AdS in [13] and it is given by:

$$
h_{\mu \nu}^{\log }=f(\tau, \rho) h_{\mu \nu}^{\mathrm{m}}
$$

with:

$$
f(\tau, \rho)=\frac{1}{2}(-2 i \tau-\log (\sinh (2 \rho))+\log (\tanh (\rho))) .
$$

Following the AdS/CFT logic we can compute two point correlation functions on the boundary at the critical point. We couple the massless mode to the stress-energy tensor $T_{i j}$ at the boundary, while the logarithmic mode is coupled to the logarithmic partner operator of the stress-energy tensor $t_{i j}$. In three dimensions the correlation functions are those of a two-dimensional LCFT [7, 8, 9]:

$$
\begin{gathered}
\left\langle T_{z z}(z, \bar{z}) T_{z z}(0)\right\rangle=0, \\
\left\langle t_{z z}(z, \bar{z}) T_{z z}(0)\right\rangle=\frac{b}{|z|^{4}}, \\
\left\langle t_{z z}(z, \bar{z}) t_{z z}(0)\right\rangle=\frac{1}{z^{4}}(-2 b \log z+\lambda),
\end{gathered}
$$

where $b=-\frac{6 \sigma l}{G_{N}}$ is the 'new anomaly'. Similar correlation functions apply for the anti-holomorphic part. These are the two-point correlators for a rank 2 LCFT 1.6-1.8 with conformal weight of two.

If we impose Brown-Henneaux boundary conditions to critical gravity, the log-modes will be truncated from the theory. The resulting theory will be trivial in the sense that it only contains null-states and the Schwarzschild-AdS black hole has zero mass. If we relax the Brown-Henneaux boundary conditions to allow for logarithmic asymptotics, the bulk theory is no longer unitary, but its dual field theory is an LCFT. One can also find black hole solutions with logarithmic asymptotics $[25,26]$ which have positive mass for the wrong sign Einstein-Hilbert term. In three dimensions this coincides with the requirement for the unitarily propagating bulk mode. 


\section{Non-Relativistic Logarithmic CFT}

In order to look for a non-relativistic scaling version of the AdS/LCFT correspondence outlined above, we work with a scalar field model which mimics critical gravity. This makes the equations much more tractable while keeping the same overall structure of the theory. The scalar fields obey a fourth order equation of motion given by:

$$
\left(\square-m_{1}^{2}\right)\left(\square-m_{2}^{2}\right) \phi=0 \text {. }
$$

This is the spin-0 analogue of equation (2.7). We study solutions of this equation in a fixed Lifshitz background (1.2). For generic masses $m_{1}^{2} \neq m_{2}^{2}$ the spectrum contains two massive spin- 0 excitations obeying second order differential equations. At the critical point $m_{1}^{2}=m_{2}^{2}$, the analogue of the critical point in NMG, the two Klein-Gordon operators in (3.1) degenerate and a logarithmic mode appears. The equations of motion at this point are the spin- 0 version of (2.9) and (2.10):

$$
\left(\square-m^{2}\right) \phi^{\mathrm{s}}=0, \quad\left(\square-m^{2}\right) \phi^{\log }=\phi^{\mathrm{s}} .
$$

The solutions can be written as:

$$
\begin{aligned}
\phi^{s}(r, x) & =\int d^{d} x^{\prime} \phi_{1(0)}\left(x^{\prime}\right) G_{K G}\left(r, x ; 0, x^{\prime}\right), \\
\phi^{\log }(r, x) & =\int d^{d} x^{\prime}\left(\phi_{2(0)}\left(x^{\prime}\right) G_{K G}\left(r, x ; 0, x^{\prime}\right)+\phi_{1(0)}\left(x^{\prime}\right) G_{\log }\left(r, x ; 0, x^{\prime}\right)\right),
\end{aligned}
$$

where $G_{K G}\left(r, x ; 0, x^{\prime}\right)$ and $G_{\log }\left(r, x ; 0, x^{\prime}\right)$ are the bulk-to-boundary propagators for the scalar and the $\log$ mode respectively. Explicit solutions for these functions for $z=2$ are given in [1], here it should suffice to remark that for the $\phi^{\log }$-mode we must relax the Fefferman-Graham near-boundary expansion to include logarithmic terms.

Correlation functions for this theory can be calculated by explicitly constructing the action which reproduces these equations of motion. Then this action may be renormalized in the holographic sense, as outlined in [27]. The correlation functions on the boundary of the Lifshitz spacetime can be seen as defining a non-relativistic scaling version of a Logarithmic Conformal Field Theory. They are in general:

$$
\begin{aligned}
\left\langle\mathscr{O}^{\mathrm{s}}\left(t_{1}, \mathbf{x}_{1}\right) \mathscr{O}^{\mathrm{s}}\left(t_{2}, \mathbf{x}_{2}\right)\right\rangle & =0, \\
\left\langle\mathscr{O}^{\log }\left(t_{1}, \mathbf{x}_{1}\right) \mathscr{O}^{\mathrm{s}}\left(t_{2}, \mathbf{x}_{2}\right)\right\rangle & =\frac{1}{\left|\mathbf{x}_{1}-\mathbf{x}_{2}\right|^{2 \Delta}} f(\chi), \\
\left\langle\mathscr{O}^{\log }\left(t_{1}, \mathbf{x}_{1}\right) \mathscr{O}^{\log }\left(t_{2}, \mathbf{x}_{2}\right)\right\rangle & =\frac{1}{\left|\mathbf{x}_{1}-\mathbf{x}_{2}\right|^{2 \Delta}}\left(-g(\chi) \log \left|\mathbf{x}_{1}-\mathbf{x}_{2}\right|+\lambda\right),
\end{aligned}
$$

with $f(\chi), g(\chi)$ functions of the scale invariant variable $\chi=\frac{\mathbf{x}^{z}}{t}$. The constant $\lambda$ is related to a wellknown shift invariance in LCFTs: $\mathscr{O}^{\log } \rightarrow \mathscr{O}^{\log }+\lambda \mathscr{O}^{\text {s }}$. This invariance is also present in the scalar field model, where one can always add to $\phi^{\log }$ solutions to its homogeneous equation of motion.

For $z=2$ we explicitly calculated the full two point functions in Fourier space. They are [1]:

$$
\left\langle\mathscr{O}_{\Delta}^{\mathrm{s}}(\omega, \mathbf{k}) \mathscr{O}_{\Delta}^{\mathrm{s}}(-\omega,-\mathbf{k})\right\rangle=0
$$




$$
\begin{aligned}
\left\langle\mathscr{O}_{\Delta}^{\mathrm{s}}(\omega, \mathbf{k}) \mathscr{O}_{\Delta}^{\log }(-\omega,-\mathbf{k})\right\rangle= & (2 \Delta-(d+1)) \omega^{\Delta-\frac{d+1}{2}} \frac{\Gamma\left(\frac{d+1}{2}-\Delta\right) \Gamma\left(\frac{|\mathbf{k}|^{2}+(2 \Delta-(d-1)) \omega}{4 \omega}\right)}{\Gamma\left(\Delta-\frac{d+1}{2}\right) \Gamma\left(\frac{|\mathbf{k}|^{2}-(2 \Delta-(d+3)) \omega}{4 \omega}\right)}, \\
\left\langle\mathscr{O}_{\Delta}^{\log }(\omega, \mathbf{k}) \mathscr{O}_{\Delta}^{\log }(-\omega,-\mathbf{k})\right\rangle= & \omega^{\Delta-\frac{d+1}{2}} \frac{\Gamma\left(\frac{d+1}{2}-\Delta\right) \Gamma\left(\frac{|\mathbf{k}|^{2}+(2 \Delta-(d-1)) \omega}{4 \omega}\right)}{\Gamma\left(\Delta-\frac{d+1}{2}\right) \Gamma\left(\frac{|\mathbf{k}|^{2}-(2 \Delta-(d+3)) \omega}{4 \omega}\right)}(\log \omega \\
& -\psi\left(\frac{d+1}{2}-\Delta\right)+\frac{1}{2} \psi\left(\frac{|\mathbf{k}|^{2}+(2 \Delta-(d-1)) \omega}{4 \omega}\right) \\
& \left.-\psi\left(\Delta-\frac{d+1}{2}\right)+\frac{1}{2} \psi\left(\frac{|\mathbf{k}|^{2}-(2 \Delta-(d+3)) \omega}{4 \omega}\right)+\lambda\right)
\end{aligned}
$$

Here $\psi(x)$ is the digamma function defined by $\psi(x)=\Gamma^{\prime}(x) / \Gamma(x)$. The correlation function (3.9) agrees with the two point function for a massive scalar field in a Lifshitz background found in [3] and later by means of holographic renormalization in [28], while the general structure is that of an LCFT with non-relativistic scale invariance.

\section{Conclusion}

The correlation functions (3.5-3.7) can be thought of as defining the non-relativistic LCFT through holographic reasoning. They agree with what one would expect from Lifshitz scaling arguments. The functions $f(\chi)$ and $g(\chi)$ can be obtained by explicitly solving the bulk field equations for a specific critical exponent $z$ and we presented the full solution for $z=2$. In the AdS limit $(z=1)$ it is shown in [1] that the correlation functions reduce to an LCFT (1.6-1.8). This agrees with earlier results found in [19].

In addition, a scalar field model for critical gravity is presented. The model is a spin- 0 analogue of the linearized equations of motion of critical gravity and can thus be useful as a toy model of this theory in general dimensions. This toy model can be useful for studying other deformations of the AdS/LCFT correspondence, such as extending it to a model of higher rank LCFTs [29]. It may also be interesting to consider non-relativistic scaling solutions of the full spin-2 theory. In this respect it is of interest to note that NGM and TMG generally allow for Lifshitz vacua.

\section{Acknowledgments}

The author would like to thank the Corfu Summer Institute for hospitality and all the participants of the Corfu Summer School for creating a very friendly and relaxed atmosphere. A special thanks goes out to Eric Bergshoeff, Jan Rosseel and Sjoerd de Haan for collaborating on the results presented at the conference.

\section{References}

[1] E. A. Bergshoeff, S. de Haan, W. Merbis, and J. Rosseel, “A Non-relativistic Logarithmic Conformal Field Theory from a Holographic Point of View,” JHEP 1109 (2011) 038, arXiv: 1106.6277 [hep-th]. 
[2] J. M. Maldacena, "The large N limit of superconformal field theories and supergravity," Adv. Theor. Math. Phys. 2 (1998) 231-252, arXiv : hep-th/9711200.

[3] S. Kachru, X. Liu, and M. Mulligan, "Gravity duals of Lifshitz-like fixed points," Phys. Rev. D 78 no. 10, (Nov, 2008) 106005.

[4] D. T. Son, "Toward an AdS/cold atoms correspondence: a geometric realization of the Schroedinger symmetry," Phys. Rev. D78 (2008) 046003, arXiv:0804.3972 [hep-th] .

[5] K. Balasubramanian and J. McGreevy, "Gravity duals for non-relativistic CFTs," Phys. Rev. Lett. 101 (2008) 061601, arXiv:0804.4053 [hep-th].

[6] D. Grumiller and N. Johansson, "Instability in cosmological topologically massive gravity at the chiral point," JHEP 07 (2008) 134, arXiv: 0805 .2610 [hep-th ] .

[7] K. Skenderis, M. Taylor, and B. C. van Rees, “Topologically Massive Gravity and the AdS/CFT Correspondence,” JHEP 09 (2009) 045, arXiv:0906.4926 [hep-th] .

[8] D. Grumiller and I. Sachs, "AdS (3) / LCFT (2) - Correlators in Cosmological Topologically Massive Gravity,” JHEP 1003 (2010) 012, arXiv:0910.5241 [hep-th] .

[9] D. Grumiller and O. Hohm, “AdS(3)/LCFT(2): Correlators in New Massive Gravity,” Phys.Lett. B686 (2010) 264-267, arXiv:0911.4274 [hep-th].

[10] H. Lu and C. N. Pope, "Critical Gravity in Four Dimensions," Phys. Rev. Lett. 106 (2011) 181302, arXiv:1101.1971 [hep-th].

[11] S. Deser et al., "Critical Points of D-Dimensional Extended Gravities," Phys. Rev. D83 (2011) 061502, arXiv:1101.4009 [hep-th].

[12] M. Alishahiha and R. Fareghbal, "D-Dimensional Log Gravity,” Phys.Rev. D83 (2011) 084052 , arXiv:1101.5891 [hep-th].

[13] E. A. Bergshoeff, O. Hohm, J. Rosseel, and P. K. Townsend, "Modes of Log Gravity," Phys. Rev. D83 (2011) 104038, arXiv:1102.4091 [hep-th].

[14] M. Porrati and M. M. Roberts, "Ghosts of Critical Gravity," Phys.Rev. D84 (2011) 024013 , arXiv:1104.0674 [hep-th].

[15] H. Lu, Y. Pang, and C. N. Pope, "Conformal Gravity and Extensions of Critical Gravity," arXiv:1106.4657 [hep-th].

[16] V. Gurarie, "Logarithmic operators in conformal field theory," Nucl.Phys. B410 (1993) 535-549, arXiv:hep-th/9303160 [hep-th].

[17] M. Flohr, "Bits and pieces in logarithmic conformal field theory," Int. J. Mod. Phys. A18 (2003) 4497-4592, arXiv: hep-th/0111228.

[18] M. R. Gaberdiel, “An Algebraic approach to logarithmic conformal field theory,” Int.J.Mod.Phys. A18 (2003) 4593-4638, arXiv: hep-th/ 0111260 [hep-th] .

[19] I. I. Kogan, "Singletons and logarithmic CFT in AdS/CFT correspondence," Phys. Lett. B458 (1999) 66-72, arXiv: hep-th/9903162.

[20] A. M. Ghezelbash, M. Khorrami, and A. Aghamohammadi, "Logarithmic conformal field theories and AdS correspondence,” Int. J. Mod. Phys. A14 (1999) 2581-2592, arXiv : hep-th/ 9807034.

[21] S. Deser, R. Jackiw, and S. Templeton, “Topologically Massive Gauge Theories,” Annals Phys. 140 (1982) 372-411. 
[22] W. Li, W. Song, and A. Strominger, "Chiral Gravity in Three Dimensions," JHEP 04 (2008) 082, arXiv:0801.4566 [hep-th].

[23] A. Maloney, W. Song, and A. Strominger, "Chiral Gravity, Log Gravity and Extremal CFT," Phys.Rev. D81 (2010) 064007, arXiv:0903.4573 [hep-th].

[24] E. A. Bergshoeff, O. Hohm, and P. K. Townsend, "Massive Gravity in Three Dimensions," Phys.Rev.Lett. 102 (2009) 201301, arXiv:0901.1766 [hep-th] .

[25] G. Clement, "Black holes with a null Killing vector in new massive gravity in three dimensions," Class.Quant.Grav. 26 (2009) 165002, arXiv:0905.0553 [hep-th] .

[26] O. Hohm and E. Tonni, "A boundary stress tensor for higher-derivative gravity in AdS and Lifshitz backgrounds," JHEP 1004 (2010) 093, arXiv:1001.3598 [hep-th].

[27] K. Skenderis, “Lecture notes on holographic renormalization,” Class. Quant. Grav. 19 (2002) 5849-5876, arXiv:hep-th/0209067.

[28] M. Taylor, "Non-relativistic holography," arXiv:0812.0530 [hep-th].

[29] E. A. Bergshoeff, S. de Haan, W. Merbis, M. Porrati, and J. Rosseel, "Unitary Truncations and Critical Gravity: a Toy Model," arXiv:1201.0449 [hep-th]. 